\title{
Long-Lived Room-Temperature Phosphorescent Nitrogen-Doped CQDs/PVA Composites: Fabrication, Characterization and Application
}

\author{
Xueyun Wu, ${ }^{1}$ Wei $\mathrm{Li},{ }^{1 *}$ Peng Wu, ${ }^{1,2}$ Chunhui Ma, ${ }^{1}$ Yushan Liu, ${ }^{1}$ Mingcong $\mathrm{Xu}^{1}$ and Shouxin Liu ${ }^{1 *}$
}

Room-temperature phosphorescent (RTP) materials have attracted significant attention because of their potential applications in anticounterfeiting and optical imaging. However, most RTP materials developed to date have short-lived lifetimes, because the triplet excited states are easily quenched by atmospheric oxygen, thus limiting their full potential. Herein, nitrogen-doped carbon quantum dots (NCQDs), prepared by hydrothermal method, are dispersed into a poly(vinyl alcohol) matrix to fabricate phosphorescent materials with ultralong lifetimes at ambient temperature and atmosphere. The N-CQDs/PVA composites, which are 3D aerogel and thin film respectively, display a long lifetime of $442 \mathrm{~ms}$ and an average lifetime of $416 \mathrm{~ms}$ at ambient conditions. The efficient room-temperature phosphorescence phenomenon can be attributed to the small energy gaps between the singlet and triplet states $\left(\Delta \mathrm{E}_{\mathrm{S}, \mathrm{T}}\right)$ of $0.246 \mathrm{eV}$ and 0.23 $\mathrm{eV}$ of the RTP materials (aerogel and thin film, respectively), thus facilitating state population through intersystem crossing. In addition, PVA molecule is able to inhibit quenching of the triplet excited state by oxygen, consequently promoting phosphorescence production at ambient conditions. Furthermore, the N-CQDs/PVA composites can be prepared in different physical forms, i.e., as solution, film, or aerogel as per the requirements of varied potential applications in optical imaging, writing, anti-counterfeiting, or sensors.

Keywords: Room-temperature phosphorescence; Long-lived lifetimes; Nitrogen-doped CQDs; Hydrothermal synthesis; PVA

Received 27 September 2018, Accepted 31 October 2018

DOI: $10.30919 / \mathrm{es} 8 \mathrm{~d} 785$

\section{Introduction}

Room-temperature phosphorescence is obtained upon excitation of triplet states via efficient intersystem crossing (ISC) of singlet to triplet and is sustained at room temperature when the excitation source is turned off. ${ }^{1}$ To date, room-temperature phosphorescent (RTP) materials with long-lived excited states have generated considerable attention because of their potential applications in advanced anti-counterfeiting, optical imaging, photocatalysis, optoelectronics, data security, and sensors. ${ }^{2-8}$ However, most of the RTP materials examined to date have been prepared using inorganic or heavy metal organic complexes, making them toxic, expensive, and unstable, ${ }^{7,9}$ as well as exhibiting short-lived lifetimes in the range of several microseconds to several milliseconds. ${ }^{10-12}$ The low phosphorescence is due to the quenching of the triplet states emission by atmospheric oxygen. ${ }^{13}$ Therefore, the preparation of low-toxic, metal-free RTP materials with ultralong lifetimes at ambient conditions is highly desired. Noteworthy, the roomtemperature phosphorescence process involves spin-forbidden transitions, which are responsible for low probability of ISC. ${ }^{14,15}$ In addition, triplet excitations are easily consumed through nonradiative

${ }^{1}$ Key laboratory of Bio-based Material Science and Technology of Ministry of Education, Northeast Forestry University, Hexing Road 26, Harbin 150040,P. R. China

${ }^{2}$ College of Environmental and Chemical Engineering, Heilongjiang University of Science and Technology, Harbin 150022, P. R. China

*E-mail: liwei19820927@126.com ; liushouxin@126.com decay processes derived from vibrational and rotational dissipation. ${ }^{14}$ Thus, effectively obtaining stable triplet states remain a great challenge. Generally, if the energy gap between the singlet and triplet levels $\left(\Delta \mathrm{E}_{\mathrm{S}, \mathrm{T}}\right)$ is small, nonradiative decay processes will be effectively suppressed, subsequently promoting highly efficient ISC and the room-temperature phosphorescence phenomenon. ${ }^{16}$ When it comes to mental-free RTP materials, the stable triplet states are typically obtained by incorporating carbonyl functionality, halogen bonds and $\mathrm{C}-\mathrm{N} / \mathrm{C}=\mathrm{N}$ bonds. ${ }^{17}$ With carbonyl functionality, the $\Delta \mathrm{E}_{\mathrm{S}, \mathrm{T}}$ is smaller in the order of $0.207 \mathrm{eV}-0.725 \mathrm{eV}$ to favour ISC process. ${ }^{18}$ In addition, $\mathrm{C}-\mathrm{N} / \mathrm{C}=\mathrm{N}$ bonds have reported to increase the population of the triplet states, which may facilitate the processes of ISC and enhance spin-orbit coupling. ${ }^{19}$ Therefore, effectively suppressing nonradiative decay processes and reducing $\Delta \mathrm{E}_{\mathrm{S}, \mathrm{T}}$ are essential to achieving long-lived excited states for RTP materials.

In recent years, carbon quantum dots (CQDs) have emerged as new luminescent nanomaterials and received considerable attention because of their various excellent properties. ${ }^{20-22}$ However, pure CQDs do not readily exhibit delayed luminescence because the triplet excited states are easily quenched by oxygen and consumed by nonradiative decay processes. Note that phosphorescence from a luminophore is typically observed under cryogenic and inert conditions because the triplet excited states are sensitive to temperature and oxygen. ${ }^{23}$ Recently, a "dots-in-zeolites" strategy has been devised for the preparation of CQD-based materials with thermally activated delayed fluorescence and ultralong lifetimes at ambient conditions. This strategy affords effective stabilization of the triplet states by restricting the intramolecular vibrations and rotations of functional groups on the CQDs and hindering oxygen 
quenching. ${ }^{24}$ Significantly, finding a suitable host matrix to harvest triplet excited states and prevent quenching at ambient conditions in obtaining CQDs with ultralong lifetime phosphorescence would be of great necessary. As an ideal candidate, poly(vinyl alcohol) (PVA) molecule has a low permeability to oxygen and abundant $\mathrm{OH}$ groups, which is beneficial for hydrogen bond formation. ${ }^{25-28}$ Generally, the direct dispersion of photoluminescent materials into polymeric materials requires organic solvents to obtain homogeneous composites. ${ }^{29}$ However, RTP materials have been successfully prepared in the absence of additional organic solvents when dispersing CQDs into PVA matrix to obtain homogeneous composites. ${ }^{30}$ Therefore, the dispersion of CQDs into PVA matrix in the absence of additional organic solvents to produce CQDs/PVA composites is possible.

Herein, hydrothermal method was used to synthesize nitrogendoped CQDs (N-CQDs) using glucose as the carbon source. The NCQDs were then dispersed into PVA matrix to construct phosphorescent materials with ultralong lifetimes at ambient temperature and atmosphere. The formation mechanism of the $\mathrm{N}$ CQDs-based RTP materials was proposed. In addition, the application of the N-CQDs/PVA composites prepared as a luminous solution, a transparent film, and a three-dimensional (3D) aerogel was investigated.

\section{Experimental section}

\subsection{Materials}

Glucose was purchased from Tianjin Kermel Chemical Reagent Co., Ltd. China. p-Aminobenzoic acid (PABA) was obtained from Tianjin Guang Fu Chemical Reagent Co., Ltd. China. Polyvinyl alcohol (PVA, average degree of polymerization, $1750 \pm 50$ ) was purchased from Aladdin Industrial Corporation (Shanghai, China). All chemical reagents were of analytical grade and used as received. Distilled water was used in all experiments.

\subsection{Preparation of N-CQDs}

For the synthesis of N-CQDs, $0.76 \mathrm{~g}$ PABA was dissolved in $60 \mathrm{~mL}$ water at $80{ }^{\circ} \mathrm{C}$ because of PABA easily dispersed in hot water. The resulting solution was transferred to a polytetrafluoroethylene vessel $(100 \mathrm{~mL})$, which contained $1 \mathrm{~g}$ glucose, and stirred slowly to make sure obtainning uniform solution. The molar ratio between PABA and glucose was $1: 1$. The vessel was sealed by an explosion-proof enclosure and heated in an oven for $12 \mathrm{~h}$ at $200{ }^{\circ} \mathrm{C}$ and subsequently allowed to cool to room temperature. The product was obtained following filtration of the precipitate through a filter membrane with pore diameters of $0.22 \mu \mathrm{m}$ and subsequent dialysis (1000 Da molecular weight cut-off) for about $72 \mathrm{~h}$ to remove unreacted small molecules, then N-CQDs powder was obtained by oven drying for further use.

2.3 Preparation of N-CQDs/PVA ink and N-CQDs/PVA film To prepare the N-CQDs/PVA composites, $1 \mathrm{mg}$ N-CQDs powder was dispersed into $1 \mathrm{~mL}$ distilled water, and then the ultrasound
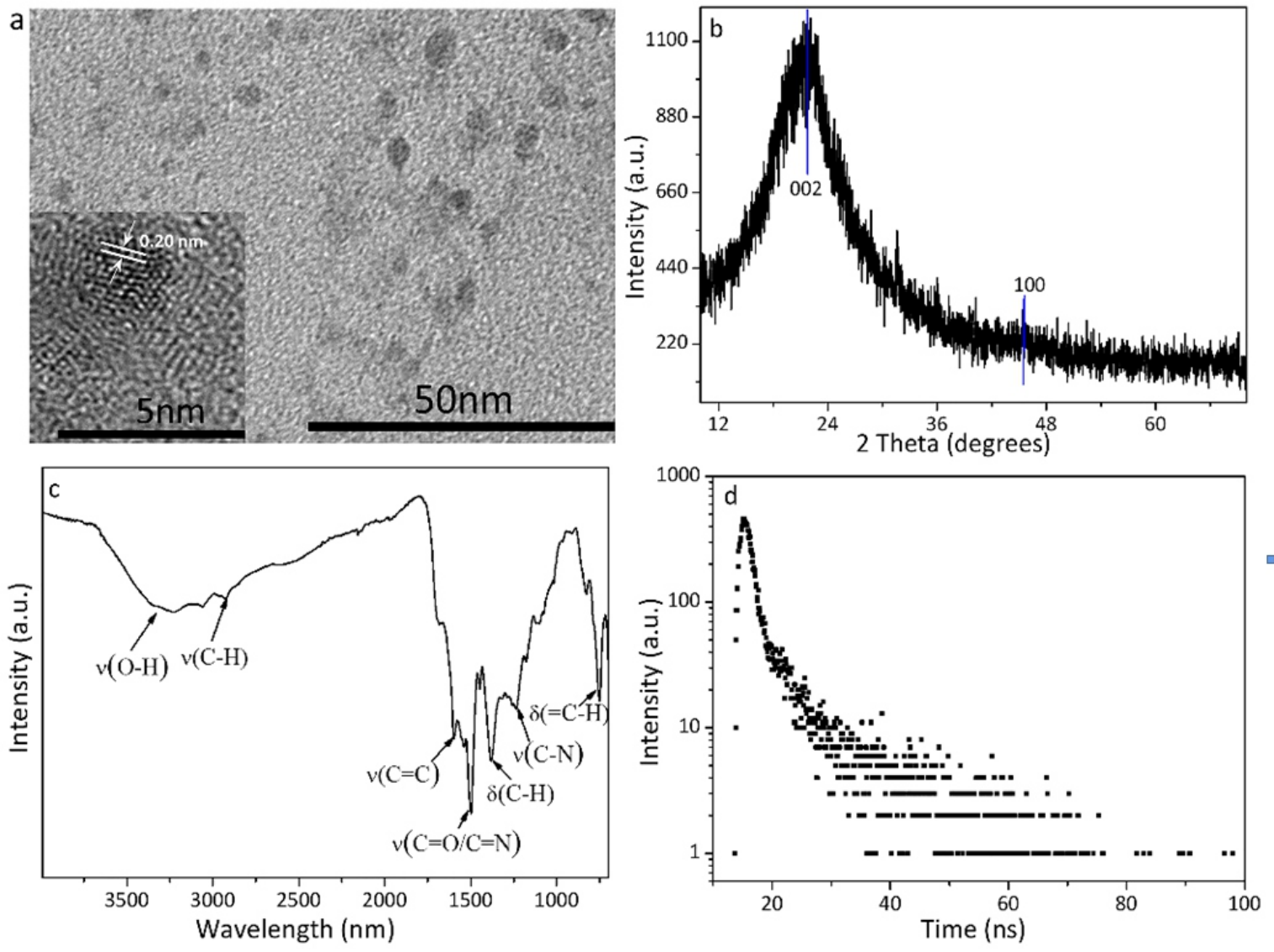

Fig. 1 (a) TEM image of the N-CQDs (inset, HRTEM image of the N-CQDs), (b) XRD pattern of the N-CQDs, (c) the FT-IR spectrum of the $\mathrm{N}-\mathrm{CQDs}$, and (d) fluorescence decay of the N-CQDs dispersed in water. 
dispersed uniformly for half hour. $1 \mathrm{~mL} \mathrm{~N}$-CQDs aqueous solution was slowly dropped into $2 \mathrm{~mL}$ PVA solution (7.5 wt.\%). The resulting mixture was stirred thoroughly to obtain a homogeneous $\mathrm{N}$ CQDs/PVA solution. The well dispersed mixture was conditioned to elimate bubbles and then could be employed as luminescent ink. Such obtained homogeneous composite solution was coated onto glass or polytetrafluoroethylene petri dish and then dried in an oven at $60{ }^{\circ} \mathrm{C}$ for hours to obtain the N-CQDs/PVA composites films.

\subsection{Preparation of spongy N-CQDs/PVA aerogel}

To prepare the composite aerogel, $10 \mathrm{mg}$ N-CQDs powder was dispersed into $10 \mathrm{~mL}$ distilled water, and then the ultrasound dispersed uniformly for half hour. The obtained N-CQDs aqueous solution was transferred into a $50 \mathrm{~mL}$ round-bottom flask and heated to $80{ }^{\circ} \mathrm{C}$ under magnetic stirring. Then, $1 \mathrm{~g}$ PVA was added to the solution, and the mixture was heated further for $2 \mathrm{~h}$ under stirring to make sure PVA dissolved completely. Finally, the obtained NCQDs/PVA solution was allowed to naturally cool to room temperature, and then precooling in the refrigerator before being subjected to freeze drying to obtain the aerogel.

\subsection{Characterizations}

A JEM-2100F electron microscope (JEOL, Ltd., Japan) operating at an acceleration voltage of $200 \mathrm{kV}$ was used to obtain transmission electron microscopy (TEM) and high-resolution TEM (HRTEM) images of the N-CQDs. The sample was ultrasonicated for $1 \mathrm{~h}$ before testing in the ice bath. The resulting dispersion was dropped onto a copper grid coated with an ultrathin carbon film and then dried at room temperature. Fourier transform infrared (FT-IR) spectra of the N-CQDs were recorded on a Nicolet iS10 FT-IR spectrometer (Thermo Scientific, USA) in the range of 500-4000 $\mathrm{cm}^{-1}$. X-ray photoelectron (XPS) spectra of the N-CQDs were obtained on a Thermo ESCALAB $250 \mathrm{Xi}$ photoelectron spectrometer (Thermo
Fisher Scientific, USA), using Al $\mathrm{K} \alpha$ radiation $(\mathrm{hv}=1486.6 \mathrm{eV})$. Binding energies were referenced to the $\mathrm{C} 1 \mathrm{~s}$ line at $284.8 \mathrm{eV}$. The UV-visible (UV-vis) absorption spectra of the N-CQDs were recorded on a TU-1950 UV-vis spectrometer (Persee, China). X-ray diffraction (XRD) patterns were obtained on a D/max-r B X-ray diffractometer (Rigaku Corp., Tokyo, Japan) using $\mathrm{Cu} \mathrm{K} \alpha$ radiation. The fluorescence emission spectra of the N-CQDs were recorded using a Hitachi F4500 fluorescence spectrophotometer (Hitachi High-Technologies, Japan). The phosphorescence emission and excitation spectra and phosphorescence decay curves of the $\mathrm{N}$ CQDs/PVA composite film and aerogel were recorded on an FLS980 fluorescence spectrophotometer (Edinburgh, UK). All digital pictures were captured using a Canon camera.

\section{Results and discussion}

\subsection{Characteristics of N-CQDs}

The TEM image in Fig. 1a reveals that the prepared N-CQDs are spherical and uniformly dispersed with minimal aggregation. In addition, the N-CQDs have a particle size distribution in the range of 1.3-6.0 nm, with a mean diameter of $3.3 \mathrm{~nm}$ (Fig. S1). Distinct lattice fringes with an interplanar spacing of $0.20 \mathrm{~nm}$ can be observed from the HRTEM image (Fig. 1a inset), which closely matches the $\left(\begin{array}{lll}1 & 0 & 0\end{array}\right)$ diffraction facet of graphite carbon. ${ }^{31}$ This result indicates that the prepared N-CQDs possess a graphite-like structure. The XRD pattern of the N-CQDs (Fig. 1b) shows a broad diffraction peak at $21.7^{\circ}\left(\mathrm{d}_{002}=0.34 \mathrm{~nm}\right)$ and a weak peak at $45.3^{\circ}\left(\mathrm{d}_{100}=0.21\right.$ $\mathrm{nm}$ ), indicating the presence of an amorphous carbon phase and partial graphitization of the N-CQDs, as consistent with the HRTEM result. $^{32}$

The FT-IR spectrum of the N-CQDs is shown in Fig. 1c. Thebroad absorption band at $3238 \mathrm{~cm}^{-1}$ can be attributed to $\mathrm{O}-\mathrm{H}$ stretching vibrations. The peak at $1600 \mathrm{~cm}^{-1}$ is attributed to aromatic $\mathrm{C}=\mathrm{C}$ stretching vibrations. ${ }^{33}$ The strong peak at $1500 \mathrm{~cm}^{-1}$ can be assigned
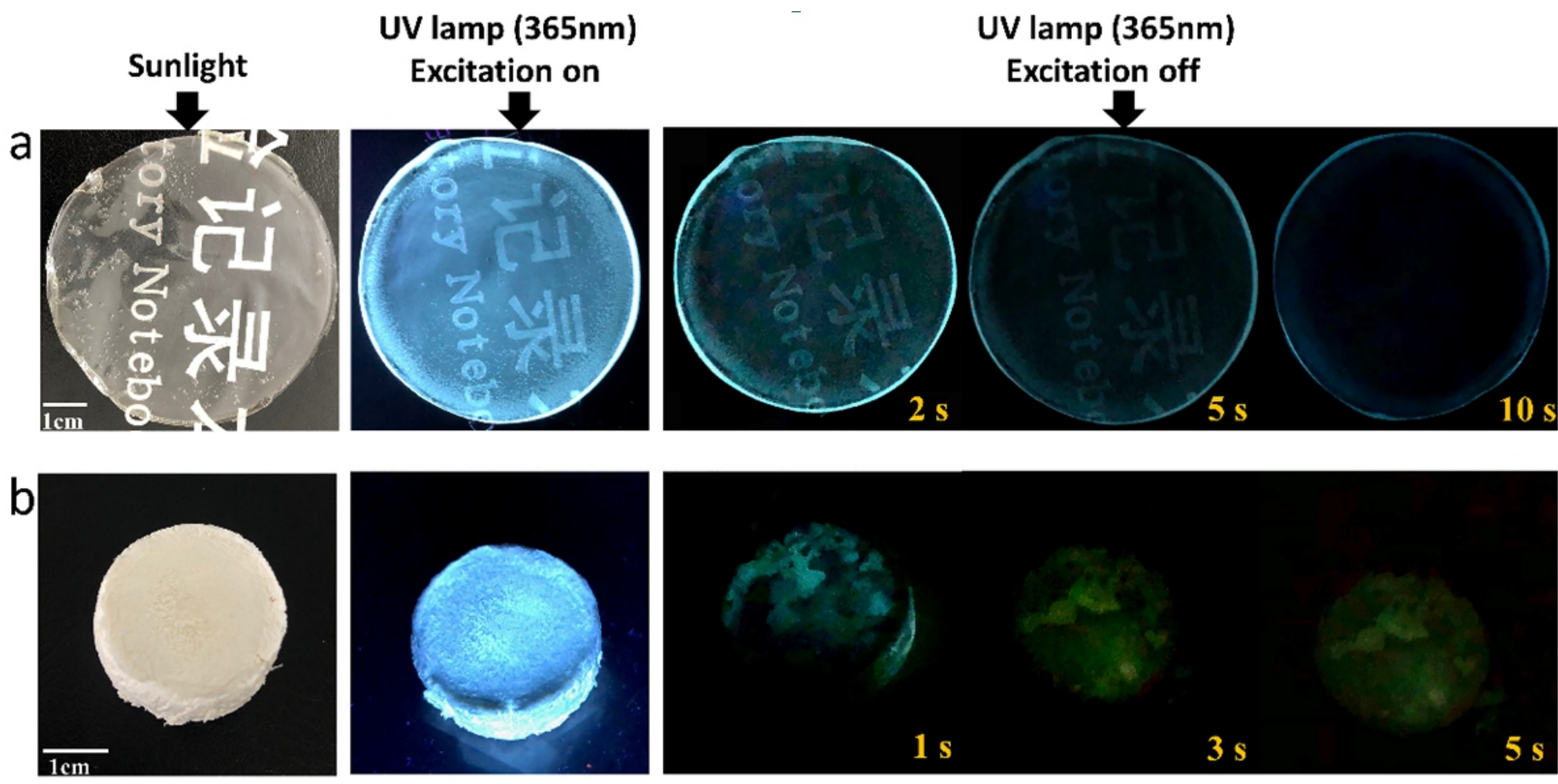

Fig. 2 Pictures of (a) N-CQDs/PVA film and (b) spongy N-CQDs/PVA composite under sunlight, UV-365 nm (ON), and after turning off the UV lamp respectively from left to right. 
to either $\mathrm{C}=\mathrm{N}$ or $\mathrm{C}=\mathrm{O}$ bond. ${ }^{34,35}$ The peaks at 2925 and $1375 \mathrm{~cm}^{-1}$ are attributed to the stretching vibrations and bending vibration of $\mathrm{C}-\mathrm{H}$ bond, respectively. ${ }^{36,37}$ The characteristic peak at $1239 \mathrm{~cm}^{-1}$ is attributed to aromatic $\mathrm{C}-\mathrm{N}$ stretching vibrations. The two peaks at 691 and $740 \mathrm{~cm}^{-1}$ can be attributed to aromatic $\mathrm{C}-\mathrm{H}$ bending vibrations. ${ }^{38}$ These results indicate that the prepared N-CQDs contain aromatic carbon and nitrogen heterocyclic rings.

To further investigate the elemental and structural composition of the N-CQDs, XPS analysis is performed, and the results are shown in Fig. S2. The full-scan XPS spectrum of the N-CQDs is shown in Fig. S2a. The high-resolution C1s spectrum of the N-CQDs (Fig. S2b) can be fitted into five peaks at binding energies of 284.7 $(\mathrm{C}-\mathrm{C}$ or $\mathrm{C}=\mathrm{C}), 286.0(\mathrm{C}-\mathrm{N}), 286.4(\mathrm{C}-\mathrm{O}), 288.0(\mathrm{C}=\mathrm{N}$ and/or $\mathrm{C}=\mathrm{O})$, and $288.4 \mathrm{eV}(\mathrm{HO}-\mathrm{C}=\mathrm{O}){ }^{39}$ The high-resolution $\mathrm{O} 1 \mathrm{~s}$ spectrum (Fig. S2c) can be deconvoluted into three peaks at $531.55(\mathrm{C}=\mathrm{O})$, $532.5(\mathrm{C}-\mathrm{OH})$, and $535.5 \mathrm{eV}\left(\mathrm{COOH}\right.$ or $\left.\mathrm{H}_{2} \mathrm{O}\right) .^{40,41}$ The $\mathrm{N} 1 \mathrm{~s}$ spectrum (Fig. S2d) can be deconvoluted into four peaks at 398.5, 399.2, 399.9 , and $400.7 \mathrm{eV}$, which confirmed the presence of nitrogen atoms of graphite-like structure, pyridinic-like $\mathrm{N}$, pyrrolic-like $\mathrm{N}$, and $\mathrm{NH}$ groups, respectively. ${ }^{42,43}$ These results confirme that the prepared N-CQDs have aromatic carbonyl and nitrogen heterocyclic rings on the surface, as consistent with the FT-IR results.

UV-vis spectrum of the N-CQDs dispersed in water is shown in Fig. S3. The prepared N-CQDs display an absorption band in the ultraviolet region owing to the presence of the aromatic $\pi$ system or the $n-\pi *$ transition of carbonyl groups. ${ }^{2,44}$ The absorption peaks centred at 240 and $350 \mathrm{~nm}$ can be ascribed to $\pi-\pi^{*}$ transition of the $\mathrm{C}=\mathrm{C}$ bond and $\mathrm{n}-\pi *$ transition of the $\mathrm{C}=\mathrm{O} / \mathrm{C}=\mathrm{N}$ bond, ${ }^{45}$ respectively. Upon excitation of the N-CQD aqueous dispersion with a hand-held UV lamp (365 nm), the dispersion displayed bright blue fluorescence (Fig. S3 inset). The fluorescence emission spectra of the N-CQDs aqueous solution are collected by varying the excitation wavelength from 300 to $460 \mathrm{~nm}$ (Fig. S4). Blue emission at $430 \mathrm{~nm}$, corresponding to the excitation wavelength of $360 \mathrm{~nm}$, can be observed. Further increases in the excitation wavelength result in the shifting of the emission peak to $460 \mathrm{~nm}$. The excitation-dependent emission of the N-CQDs may originate from the presence of different surface states. ${ }^{46}$ The fluorescence emission decay curve of the aqueous dispersion of N-CQDs is reported in Fig. 1d. The NCQDs exhibited double exponential decay with an average lifetime of $4.93 \mathrm{~ns}$. The decay parameters are summarized in Table S1.

\subsection{Photoluminescent properties of N-CQDs/PVA film and aerogel}

The N-CQDs/PVA film is transparent and nearly colourless under sunlight. In contrast, when it is irradiated with a hand-held UV lamp (365 nm), the film turned blue. Interestingly, when the excitation source is turned off, a blue-green afterglow can be seen by the naked eyes at room temperature (Fig. 2a). Fig. 2b shows the white appearance of the N-CQDs/PVA aerogel under sunlight. Its blue appearance could be observed upon exposure to a hand-held UV lamp at ambient temperature and atmosphere and a blue-green afterglow
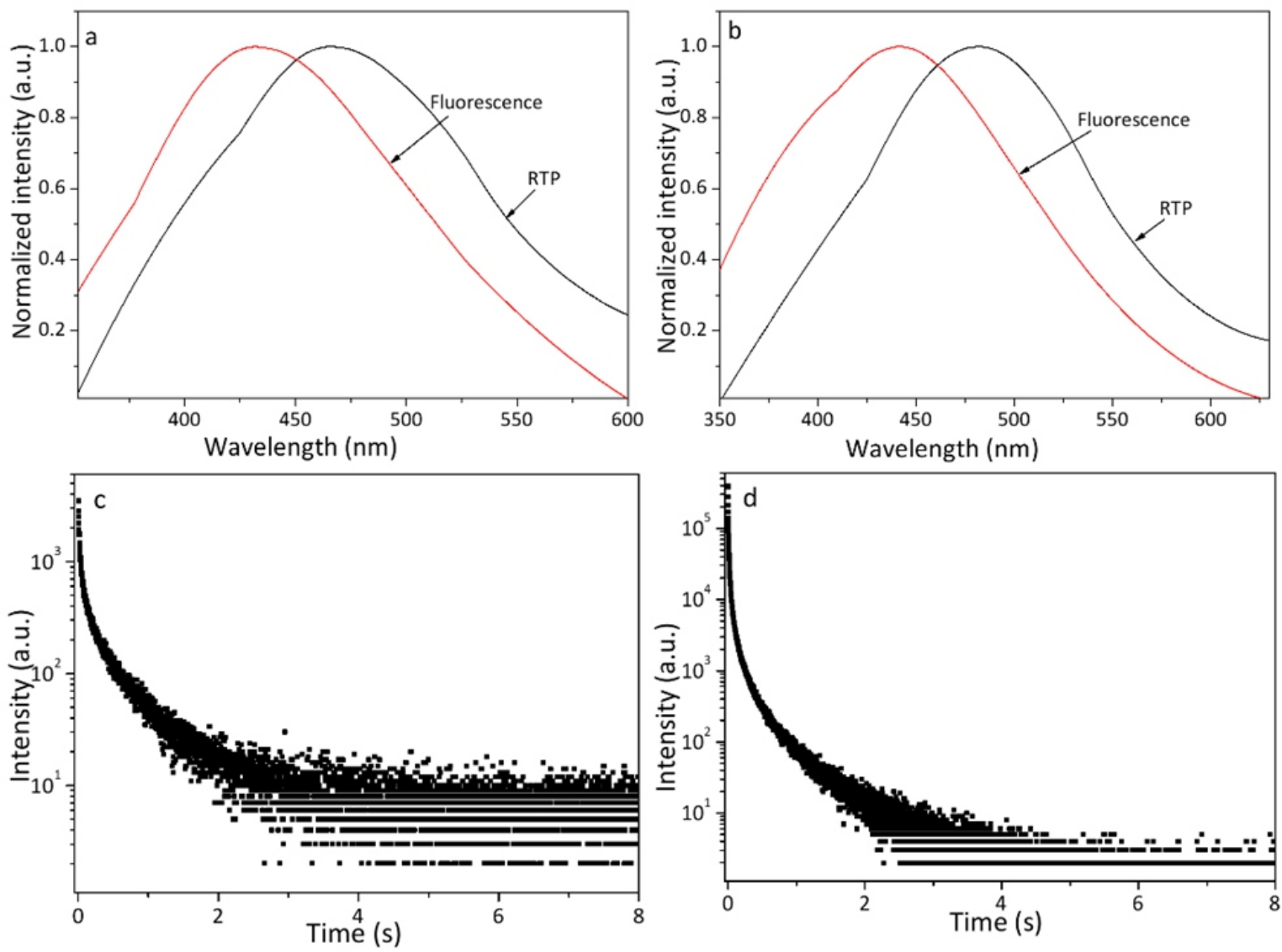

Fig. 3 Spectra of fluorescence emission and RTP emission on (a) N-CQDs/PVA film, and (b) N-CQDs/PVA aerogel; Time resolved phosphorescence decay of (c) N-CQDs/PVA film, and (d) N-CQDs/PVA aerogel. 
could be observed when the lamp was turned off.

To further explore the origin of the room-temperature phosphorescence phenomenon of the N-CQDs/PVA composites, both their steady-state fluorescence and phosphorescence photoluminescence (PL) spectrum are recorded. Fig. 3a shows the fluorescence PL spectrum and phosphorescence PL spectrum of the N-CQDs/PVA film. The film display emission at about $430 \mathrm{~nm}$ in accordance with aqueous N-CQDs (Fig. S4), and the phosphorescence PL spectrum show an emission band at $468 \mathrm{~nm}$. The Stokes shift between the fluorescence and phosphorescence is $38 \mathrm{~nm}$, corresponding to an energy gap of $0.23 \mathrm{eV}$.

The fluorescence PL spectrum of the N-CQDs/PVA aerogel displayed an emission band at $440 \mathrm{~nm}$, which can be assigned to the emission of a singlet excited state (Fig. 3b). The phosphorescence PL spectrum of the N-CQDs/PVA aerogel displayed an emission band at $482 \mathrm{~nm}$ (emission of a triplet excited state). From these results, an $\Delta \mathrm{E}_{\mathrm{S}, \mathrm{T}}$ of $42 \mathrm{~nm}$ is obtained. Accordingly, the N-CQDs/PVA composites display a small $\Delta \mathrm{E}_{\mathrm{S}, \mathrm{T}}$ of $0.246 \mathrm{eV}$ or $0.23 \mathrm{eV}$ depending on their structure (aerogel or film). The small $\Delta \mathrm{E}_{\mathrm{S}, \mathrm{T}}$ is expected to facilitate ISC from singlet state $\left(\mathrm{S}_{1}\right)$ to triplet state $\left(\mathrm{T}_{1}\right)$ state, thus facilitating the occurrence of RTP. According to literature reports, ${ }^{47-49}$ aromatic carbonyl groups display a small $\Delta \mathrm{E}_{\mathrm{S}, \mathrm{T}}$, and the spin-orbit coupling is efficient, which is beneficial for the occurrence of ISC. The role of $\mathrm{C}=\mathrm{N}$ functionalities is also reported to increase the population of the $T_{1}$ state, demonstrating that the presence of nitrogen atoms may favour ISC and thereby enhance spin-orbit coupling. ${ }^{50}$ Therefore, it is reasonable to propose that the room temperature phosphorescence phenomenon originates from aromatic carbonyl and $\mathrm{C}=\mathrm{N}$ functionalities on the surface of the N-CQDs.

The time-resolved phosphorescence decay curves of the NCQDs/PVA film and aerogel are shown in Fig. 3c and Fig. 3d, respectively. The two curves can be fitted by a double exponential function with two lifetimes. The N-CQDs/PVA film display two phosphorescence lifetimes of $185.84 \mathrm{~ms}(46.45 \%)$ and $491.45 \mathrm{~ms}$ (53.5 5\%). The N-CQDs/PVA aerogel also display two phosphorescence lifetimes of $124.83 \mathrm{~ms}(48.08 \%)$ and $513.32 \mathrm{~ms}$ $(51.92 \%)$. The average lifetimes of the film and aerogel are respectively calculated as 416 and $442 \mathrm{~ms}$ using eqn (1) as follows:

$$
<\tau>=\sum \alpha_{\mathrm{i}} \tau_{\mathrm{i}}^{2} / \sum \alpha_{\mathrm{i}} \tau_{\mathrm{i}}
$$

Moreover, the multiple lifetimes of the N-CQDs/PVA composites indicate that various emission species are present, which may be due to the different chemical environments present on the surface of the N-CQDs. Furthermore, the average lifetimes of the composites are much longer than the fluorescence lifetime $(4.93 \mathrm{~ns})$ of the prepared $\mathrm{N}-\mathrm{CQD}$ s solution at room temperature (Fig. 1d). This result suggests that the PVA matrix plays an important role in stabilizing the triplet states.

When the N-CQDs are only dispersed in water, the room temperature phosphorescence phenomenon was not observed. This may be due to the consumption of the triplet excitations by vibrational and rotational dissipation and oxygen-mediated quenching at room temperature. In contrast, the long lifetimes and sustained afterglow of the film and aerogel composites may be attributed

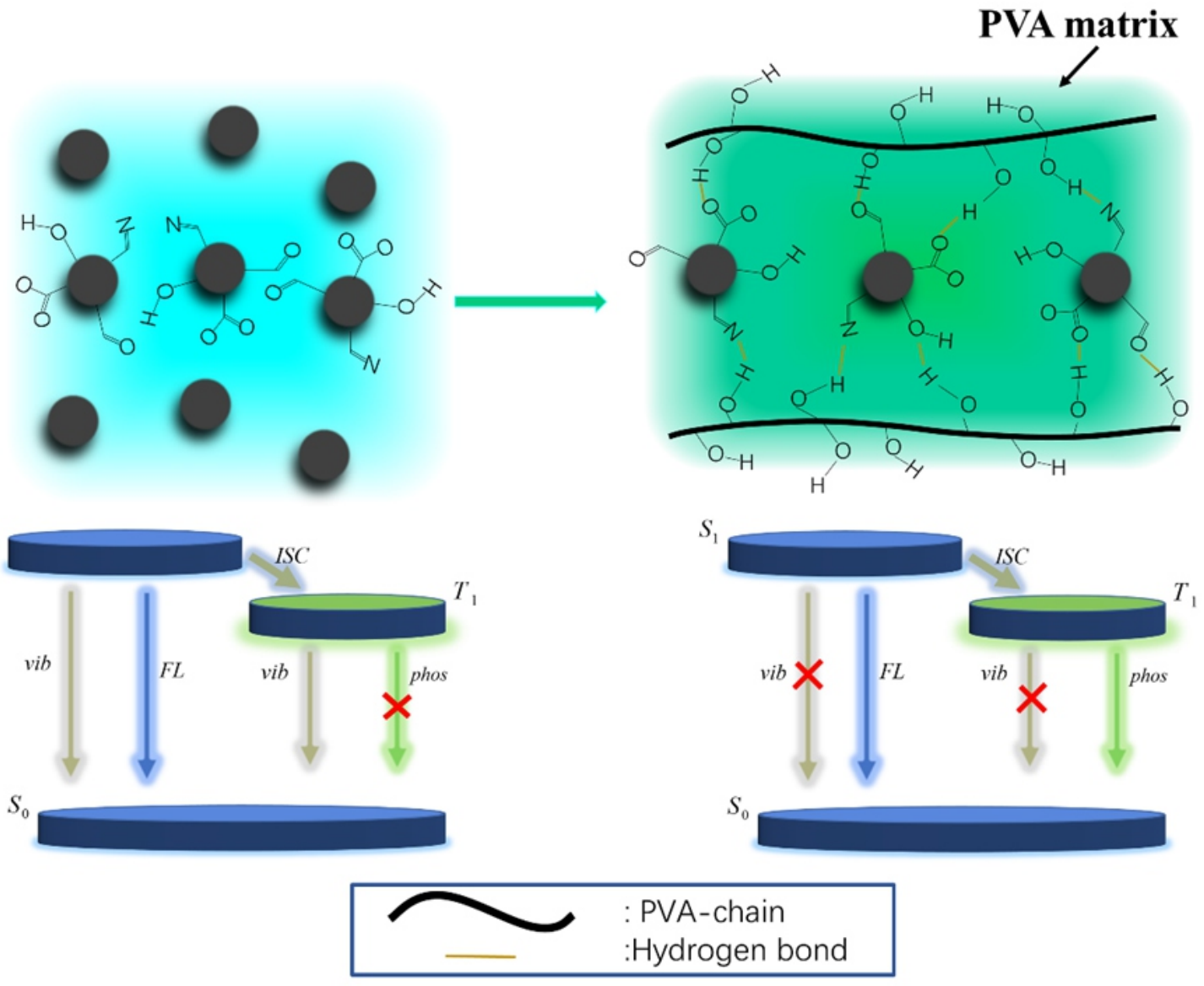

Fig. 4 Proposed phosphorescence mechanism of N-CQDs dispersed in the PVA matrix. 
to the this may be due to the consumption of the triplet excitations by vibrational and rotational dissipation and oxygen-mediated quenching at room temperature. In contrast, the long lifetimes and sustained afterglow of the film and aerogel composites may be attributed to the presence of the PVA matrix that facilitated the occurrence of RTP. It is well known that hydrogen bonding can effectively suppress intramolecular motions and fix emissive species to obtain a stable triplet state, thus favouring the ISC process. ${ }^{18}$ Thus, the abundant $\mathrm{OH}$ groups on the PVA matrix, ${ }^{28,51}$ which can form hydrogen bonds interactions with the surface functional groups of the $\mathrm{N}$-CQDs, such as $\mathrm{C}=\mathrm{N}$ and $\mathrm{C}=\mathrm{O}$ bonds, are expected to suppress intramolecular motions and nonradiative relaxation. In addition, PVA has a low permeability to oxygen, thus limiting quenching of the triplet excited states by oxygen. ${ }^{26}$ Therefore, PVA may play a key role in stabilizing the triplet excited states and serving as an excellent oxygen barrier to hinder oxygen-mediated quenching.

In RTP materials, the energy levels of the singlet and triplet states are essential considerations for efficient transition and intersystem crossing. As discussed previously, the N-CQDs/PVA composites all displayed small $\Delta \mathrm{E}_{\mathrm{S}, \mathrm{T}}$ values $(0.227 \mathrm{eV}$ and $0.246 \mathrm{eV})$, which afforded efficient room-temperature phosphorescence facilitated by ISC. A possible mechanism for the formation of RTP materials based on N-CQDs is schematically proposed in Fig. 4. The $\mathrm{N}-\mathrm{CQDs}$ dispersed in water exhibit short-lived fluorescence; in addition, in this scenario, the triplet state may be quenched by intramolecular vibrations and rotations. In contrast, mixing N-CQDs with PVA, which have abundant surface $\mathrm{OH}$ groups, produces efficient RTP materials. The abundant $\mathrm{OH}$ groups are expected to form extensive hydrogen bonds that can rigidify various functional groups on the surface of the N-CQDs to suppress the nonradiative relaxation of the N-CQDs derived from intramolecular vibrations and rotations and thus stabilize the triplet excited states. ${ }^{18}$ Moreover, PVA is an excellent oxygen barrier that can effectively hinder collisions between functional groups and oxygen molecules.

\subsection{Applications of N-CQDs/PVA composites}

The N-CQDs/PVA composites developed herein are expected to offer new perspectives for various RTP applications. Both PVA and the prepared N-CQDs have excellent biocompatible features and can be easily attached onto glass, plastic, or paper. For instance, the NCQDs/PVA composites can be printed on various packages of merchandise for authentication and used for information encryption and anti-counterfeiting. As shown in Fig. 5a, the N-CQDs/PVA solution could be used as ink - "NEFU" is directly written on a filter paper. After thorough drying, the writing can barely be seen under sunlight. However, upon irradiation with a UV lamp (365 nm), the writing is visible in blue, and when the UV source is turned off, the writing can be seen in the dark by the naked eyes as a phosphorescent blue-green colour. In another example, the outline of a bunch of grapes is "coded": N-CQDs/PVA is used to code the grapes and a fluorescent orange dye is used to code the leaves (Fig. 5b). Under sunlight, the grapes are barely visible, whereas the leaves display a very light pink tinge. In contrast, under UV exposure, the grapes and leaves can be respectively observed as blue and orange objects. When the UV source is turned off, only a phosphorescent blue-green colour, from the grapes, can be observed by the naked eyes in the dark. This application

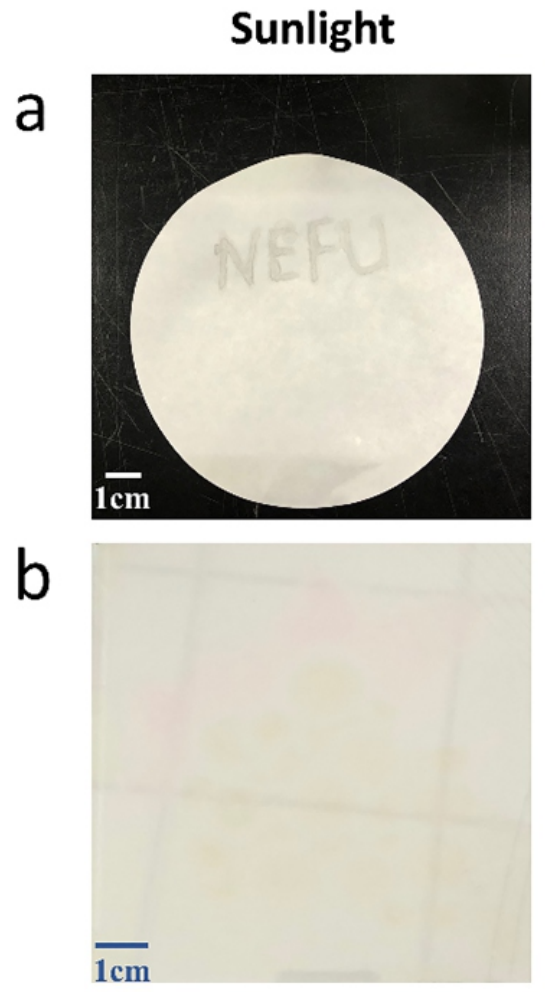

\section{UV lamp (365nm) Excitation on}
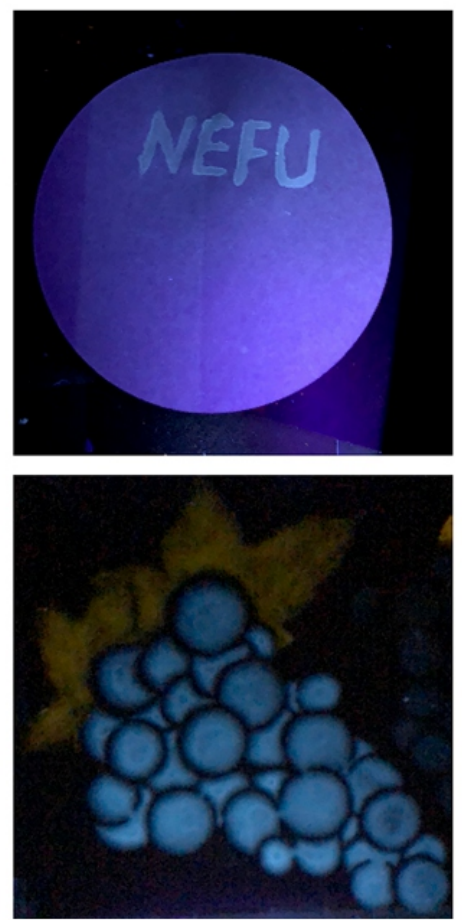

UV lamp (365nm) Excitation off
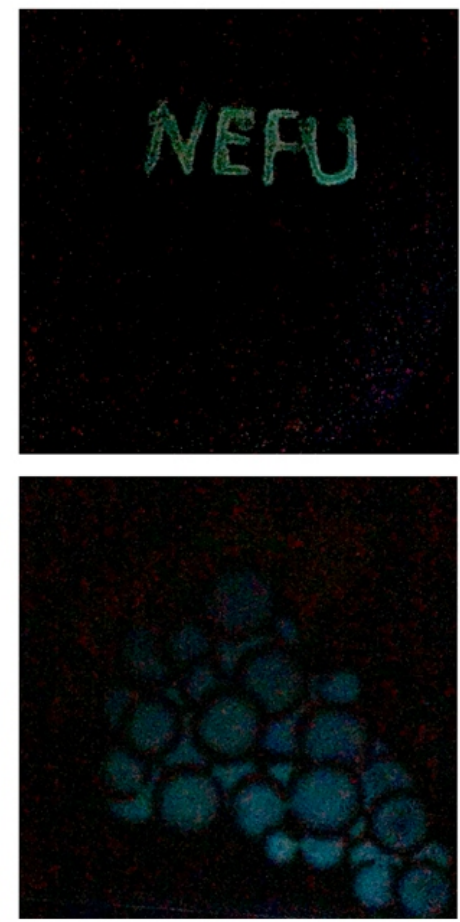

Fig. 5 (a) Handwritten characters "NEFU" on a filter paper using N-CQDs/PVA solution as ink, (b) The security pattern of a bunch of grapes were coded at glass with the N-CQDs/PVA solution as the grape grain part and the fluorescent orange dye molecule as the part of grape leaves, under sunlight, UV-365 nm (ON), and after turning off the UV lamp respectively. 
illustration demonstrates the potential of N-CQDs/PVA composites as an optical material for PL ink information security.

Moreover, the N-CQDs/PVA composites can be shaped into a film and an aerogel (Fig. S5a and Fig. S5b, respectively). Fig. S5a shows five different transparent films prepared using different materials (N-CQDs/PVA solution, pure PVA solution, and different metal quantum dots) under sunlight. Upon exposure of the films to irradiation of a UV lamp, the films display five different colours depending on the material that they were made of. When the lamp is turned off, only the film that is prepared from a N-CQDs/PVA solution exhibited a blue-green afterglow. Spongy aerogels have been well researched in the literature; they exhibit a unique porous structure that allows the absorption of different substances (e.g., carbon dioxide, oxygen) or the inhibition of aggregation or stacking of subunits to expose more active sites for photocatalytic reactions. Thus, the prepared aerogel shown in Fig. S5b is also expected to have great potential, for instance, as chemical sensors or metal-free catalysts.

\section{Conclusion}

Room-temperature phosphorescent N-CQDs/PVA composites with long-lived lifetimes are successfully fabricated by dispersing hydrothermally prepared N-CQDs into a PVA matrix. The NCQDs/PVA composites exhibited an average lifetime of $416 \mathrm{~ms}$ and a long lifetime of $442 \mathrm{~ms}$, and $\Delta \mathrm{E}_{\mathrm{s}, \mathrm{T}}$ of 0.23 and $0.246 \mathrm{eV}$, depending on the physical form of the composite (film or aerogel). The longlived excited state of the N-CQDs/PVA composites at ambient conditions is due to the small $\Delta \mathrm{E}_{\mathrm{S}, \mathrm{T}}$, which allowed population of the states through ISC. In addition, the abundant $\mathrm{OH}$ groups and low oxygen permeability of PVA matrix contributed to inhibiting quenching of the triplet excited states by nonradiative relaxation and intramolecular motions at ambient condition. Furthermore, the NCQDs/PVA solution show great performance in optical imaging and anti-counterfeiting. The ability to also shape the N-CQDs/PVA composites into a film or an aerogel offers further extensive application prospects.

\section{Supporting Information}

Additional Table S1, and additional Figures S1-S5 are provided.

\section{Conflict of interest}

The authors declare that they have no conflict of interest.

\section{Acknowledgement}

This work was supported by the National Natural Science Foundation of China (Nos. 31570567, 31500467), Fundamental Research Funds for the Central Universities of China (Nos. 2572017ET02).

\section{References}

1. K. Van den Eeckhout, P. F. Smet and D. Poelman, Materials, 2010, 3, 2536-2566.

2. K. Jiang, L. Zhang, J. Lu, C. Xu, C. Cai and H. Lin, Angew. Chem. Int. Ed., 2016, 55, 7231-7235.

3. C. Murawski, K. Leo and M. C. Gather, Adv. Mater., 2013, 25, 6801-6827.

4. L. Xu, Y. Li, S. Li, R. Hu, A. Qin, B. Z. Tang and B. Su, Analyst, 2014, 139, 2332-2335.
5. L. Bai, N. Xue, Y. Zhao, X. Wang, C. Lu and W. Shi, Nano Res., 2018, 11, 2034-2045.

6. Z. An, C. Zheng, Y. Tao, R. Chen, H. Shi, T. Chen, Z. Wang, H. Li, R. Deng, X. Liu and W. Huang, Nat. Mater., 2015, 14, 685690.

7. A. F. Henwood and E. Zysman-Colman, Chem. Commun. (Camb), 2017, 53, 807-826.

8. W. P. To, K. T. Chan, G. S. Tong, C. Ma, W. M. Kwok, X. Guan, K. H. Low and C. M. Che, Angew. Chem. Int. Ed., 2013, 52, 6648-6652.

9. E. Sotelo-Gonzalez, L. Roces, S. Garcia-Granda, M. T. Fernandez-Arguelles, J. M. Costa-Fernandez and A. Sanz-Medel, Nanoscale, 2013, 5, 9156-9161.

10. G. Zhang, J. Chen, S. J. Payne, S. E. Kooi, J. N. Demas and C. L. Fraser, J. Am. Chem. Soc., 2007, 129, 8942-8943.

11. S. Y. Lee, T. Yasuda, Y. S. Yang, Q. Zhang and C. Adachi, Angew. Chem. Int. Ed., 2014, 53, 6402-6406.

12. Q. Zhang, J. Li, K. Shizu, S. Huang, S. Hirata, H. Miyazaki and C. Adachi, J. Am. Chem. Soc., 2012, 134, 14706-14709.

13. G. Xie, X. Li, D. Chen, Z. Wang, X. Cai, D. Chen, Y. Li, K. Liu, Y. Cao and S.-J. Su, Adv. Mater, 2016, 28, 181-187.

14. S. Mukherjee and P. Thilagar, Chem. Commun. (Camb), 2015, 51, 10988-11003.

15. Y. Chen, J. He, C. Hu, H. Zhang, B. Lei and Y. Liu, J. Mater. Chem. C, 2017, 5, 6243-6250.

16. J. Joseph and A. A. Anappara, Phys. Chem. Chem. Phys., 2017, 19, 15137-15144.

17. W. Zhao, Z. He, Jacky W. Y. Lam, Q. Peng, H. Ma, Z. Shuai, G. Bai, J. Hao and Ben Z. Tang, Chem., 2016, 1, 592-602.

18. W. Z. Yuan, X. Y. Shen, H. Zhao, J. W. Y. Lam, L. Tang, P. Lu, C. Wang, Y. Liu, Z. Wang, Q. Zheng, J. Z. Sun, Y. Ma and B. Z. Tang, J. Phys. Chem. C, 2010, 114, 6090-6099.

19. M. Shimizu, R. Shigitani, M. Nakatani, K. Kuwabara, Y. Miyake, K. Tajima, H. Sakai and T. Hasobe, J. Phys. Chem. C, 2016, 120, 11631-11639.

20. L. Cao, X. Wang, M. J. Meziani, F. Lu, H. Wang, P. G. Luo, Y. Lin, B. A. Harruff, L. M. Veca, D. Murray, S. Y. Xie and Y. P. Sun, J. Am. Chem. Soc., 2007, 129, 11318-11319.

21. Y. Fang, S. Guo, D. Li, C. Zhu, W. Ren, S. Dong and E. Wang, ACS Nano, 2012, 6, 400-409.

22. W. Li, Z. Zhang, B. Kong, S. Feng, J. Wang, L. Wang, J. Yang, F. Zhang, P. Wu and D. Zhao, Angew. Chem. Int. Ed., 2013, 52, 8151-8155.

23. S. Menning, M. Kramer, B. A. Coombs, F. Rominger, A. Beeby, A. Dreuw and U. H. Bunz, J. Am. Chem. Soc., 2013, 135, 21602163.

24. J. Liu, N. Wang, Y. Yu, Y. Yan, H. Zhang, J. Li and J. Yu, Sci. $A d v ., 2017$, 3, e1603171.

25. J. He, Y. He, Y. Chen, X. Zhang, C. Hu, J. Zhuang, B. Lei and Y. Liu, Chem. Eng. J., 2018, 347, 505-513.

26. J. Gaume, P. Wong-Wah-Chung, A. Rivaton, S. Thérias and J.L. Gardette, RSC Adv., 2011, 1, 1471.

27. O. C. Compton, S. W. Cranford, K. W. Putz, Z. An, L. C. Brinson, M. J. Buehler and S. T. Nguyen, ACS Nano, 2012, 6, 2008-2019.

28. J. Bonilla, E. Fortunati, L. Atarés, A. Chiralt and J. M. Kenny, Food Hydrocolloids, 2014, 35, 463-470.

29. D. Mosconi, D. Mazzier, S. Silvestrini, A. Privitera, C. Marega, L. Franco and A. Moretto, ACS Nano, 2015, 9, 4156-4164.

30. Y. Deng, D. Zhao, X. Chen, F. Wang, H. Song and D. Shen, 
Chem. Commun. (Camb), 2013, 49, 5751-5753.

31. X. Guo, C. F. Wang, Z. Y. Yu, L. Chen and S. Chen, Chem. Commun. (Camb), 2012, 48, 2692-2694.

32. Y. Dong, N. Zhou, X. Lin, J. Lin, Y. Chi and G. Chen, Chem. Mater., 2010, 22, 5895-5899.

33. J. Jeong, J. Jung, M. Choi, J. W. Kim, S. J. Chung, S. Lim, H. Lee and B. H. Chung, Adv. Mater., 2012, 24, 1999-2003.

34. H. Li, X. He, Z. Kang, H. Huang, Y. Liu, J. Liu, S. Lian, C. H. Tsang, X. Yang and S. T. Lee, Angew. Chem. Int. Ed., 2010, 49, 4430-4434.

35. Y. Liu, Z. Chen, W. Li, C. Ma, P. Wu, X. Wu, S. Li and S. Liu, Mikrochim. Acta, 2018, 185, 470.

36. P. Wu, W. Li, Q. Wu, Y. Liu and S. Liu, RSC Adv., 2017, 7, 44144-44153.

37. H. Gu, H. Zhang, J. Lin, Q. Shao, D. P. Young, L. Sun, T. D. Shen and Z. Guo, Polymer, 2018, 143, 324-330.

38. H. Li, Z. Kang, Y. Liu and S.-T. Lee, J. Mater. Chem., 2012, 22 , 24230-24253.

39. Y. Liu, W. Li, P. Wu, C. Ma, X. Wu, M. Xu, S. Luo, Z. Xu and S. Liu, Sens. Actuators, B, 2019, 281, 34-43.

40. J. Tan, R. Zou, J. Zhang, W. Li, L. Zhang and D. Yue, Nanoscale, 2016, 8, 4742-4747.

41. H. Gu, C. Ma, C. Liang, X. Meng, J. Gu and Z. Guo, J. Mater. Chem. C, 2017, 5, 4275-4285.
42. P. Ayala, R. Arenal, A. Loiseau, A. Rubio and T. Pichler, Rev. Mod. Phys., 2010, 82, 1843-1885.

43. G. Ćirić-Marjanović, I. Pašti and S. Mentus, Prog. Mater Sci., 2015, 69, 61-182.

44. G. Eda, Y. Y. Lin, C. Mattevi, H. Yamaguchi, H. A. Chen, I. S. Chen, C. W. Chen and M. Chhowalla, Adv. Mater, 2010, 22, 505-509.

45. P. Zhang, X. Zhao, Y. Ji, Z. Ouyang, X. Wen, J. Li, Z. Su and G. Wei, J. Mater. Chem. B, 2015, 3, 2487-2496.

46. M. Goumri, J. W. Venturini, A. Bakour, M. Khenfouch and M. Baitoul, Appl. Phys. A, 2016, 122, 1-8.

47. X. Xiong, F. Song, J. Wang, Y. Zhang, Y. Xue, L. Sun, N. Jiang, P. Gao, L. Tian and X. Peng, J. Am. Chem. Soc., 2014, 136, 9590-9597.

48. O. Bolton, K. Lee, H. J. Kim, K. Y. Lin and J. Kim, Nat. Chem., 2011, 3, 205-210.

49. D. Yan, A. Delori, G. O. Lloyd, T. Friscic, G. M. Day, W. Jones, J. Lu, M. Wei, D. G. Evans and X. Duan, Angew. Chem. Int. Ed., 2011, 50, 12483-12486.

50. Y. Dai, H. Long, X. Wang, Y. Wang, Q. Gu, W. Jiang, Y. Wang, C. Li, T. H. Zeng, Y. Sun and J. Zeng, Particle \& Part. Part. Syst. Char., 2014, 31, 597-604.

51. H. A. Al-Attar and A. P. Monkman, Adv. Funct. Mater., 2012, 22, 3824-3832. 